\title{
Psychological impact of lymphoma on adolescents and young adults: not a matter of black or white
}

\author{
F. M. Drost ${ }^{1}$ - F. Mols ${ }^{1,2}$ - S. E. J. Kaal ${ }^{3}$ - W. B. C. Stevens ${ }^{4}$ - W. T. A. van der Graaf ${ }^{3,5}$. $^{2}$ \\ J. B. Prins ${ }^{6}$ • O. Husson ${ }^{6}$
}

Received: 17 November 2015 / Accepted: 20 January 2016/Published online: 8 February 2016

(C) The Author(s) 2016. This article is published with open access at Springerlink.com

\begin{abstract}
Purpose The purpose of the study is to examine differences in perceived impact of cancer (IOC) between adolescents and young adults (AYAs; $18-35$ years at cancer diagnosis), adults (36-64 years) and elderly (65-84 years) with a history of (non-)Hodgkin lymphoma. Furthermore, to investigate the association of socio-demographic, clinical and psychological characteristics with IOC; and the association between IOC and health-related quality of life (HRQoL) among AYAs only. Methods This study is part of a population-based PROFILES registry survey among lymphoma patients diagnosed between 1999 and 2009. Patients $(n=1.281)$ were invited to complete
\end{abstract}

This manuscript has been prepared in accordance with the style of the journal, and all authors have approved its contents. This manuscript is not being considered for publication elsewhere and the findings of this manuscript have not been previously published.

O. Husson

Olga.Husson@radboudumc.nl

1 CoRPS - Centre of Research on Psychology in Somatic Diseases, Department of Medical and Clinical Psychology, Tilburg University, Tilburg, The Netherlands

2 Comprehensive Cancer Centre the Netherlands (CCCN), Netherlands Cancer Registry, Eindhoven, The Netherlands

3 Department of Medical Oncology, Radboud University Medical Centre, Nijmegen, The Netherlands

4 Department of Haematology, Radboud University Medical Centre, Nijmegen, The Netherlands

5 The Institute of Cancer Research and Royal Marsden NHS Foundation Trust, London, UK

6 Department of Medical Psychology, Radboud University Medical Centre, PO Box 9101, 6500 HB Nijmegen, The Netherlands the IOCv1 and EORTC-QLQ-C30 questionnaires. Response rate was $67 \%(n=861)$.

Results AYA lymphoma survivors scored higher on the positive IOC summary scale, compared to adult and elderly patients $(p<0.001)$, while no significant differences were observed for negative IOC. Among AYAs, females, survivors with a partner, and survivors with elevated psychological distress levels scored significantly higher on the negative IOC summary scale. The negative IOC summary scale was negatively associated with all EORTC QLQ-C30 functioning scales $(\beta$ ranging from -0.39 to $-0.063 ; p<0.05$ ). The positive IOC summary scale was negatively associated with the EORTC QLQ-C30 subscale 'Emotional functioning' $(\beta=-0.24 ; p<0.05)$.

Conclusion AYA, adult and elderly with a history of (non-)Hodgkin lymphoma experienced different types of IOC in terms of positive and negative aspects.

Implications for Cancer Survivors Although AYAs experience a more positive IOC compared to older survivors, some AYAs experience more negative IOC and may require developmentally appropriate interventions to address their specific concerns.

Keywords Adolescent and young adult oncology · Impact of cancer - Lymphoma Health-related quality of life

\section{Introduction}

In The Netherlands, each year approximately 4000 people are diagnosed with non-Hodgkin lymphoma (NHL) and 400 with Hodgkin lymphoma (HL) [1]. Advances in early cancer detection, diagnosis, and treatments have noticeably improved survival rates of patients with lymphoma [2-4]. The 5-year survival rates range from $48-76 \%$ (NHL) to $95 \%$ (HL), 
depending on type and stage of tumour, treatment, and age of the patient [1]. In 2013, there were approximately 5800 patients living with a history of HL and 30,000 with NHL [1].

As the survival rate is increasing, attention is more and more given to the long-term consequences (e.g. fatigue, depression) and late effects (e.g. infertility and second cancers) of cancer and its treatment. These effects can have a negative influence on health-related quality of life (HRQoL) [5-8]. In addition, cancer survivors also perceive specific positive and negative impact of cancer (IOC) on their lives. In a positive way, cancer survivors can develop more empathy and health awareness, or a positive self-evaluation after their cancer [9]. On the other hand, cancer can influence life in a negative way, by appearance and body change concerns, and by worrying [9]. Development of the IOC questionnaire was spurred by the need to measure unique aspects of survivorship not addressed by existing HRQoL measures [9]. Whereas HRQoL measures generally focus on physical, cognitive, social and emotional functioning, the IOC measure addresses the unique issues, problems and changes that long-term survivors ascribe to their cancer experience (e.g. the survivors 'health awareness', the 'meaning of cancer' and how the experience changed the survivor) [9].

IOC may be age-specific $[5,10,11]$. Each phase in life is characterized by developmental milestones or typical life concerns [12]. For example, elderly people ( $>65$ years at diagnosis) generally have to deal with a decline in physical functioning, less social support, isolation and comorbid conditions [13-15]. In contrast, adults (36-64 years at diagnosis) more often have concerns about unemployment, financial issues and family matters $[16,17]$, while adolescents and young adults (AYAs; $18-35$ years at cancer diagnosis) are in a challenging period where they face major life tasks, such as establishing their personal identity, creating intimate relationships, gaining independence and starting careers and families [18-20]. A cancer diagnosis at AYA age can significantly disrupt or delay achieving these milestones [21]. Understanding age-related differences in IOC can contribute to the development of age-appropriate psychological interventions and personalized care. Therefore, the primary aim of the present study was to compare the IOC between AYA, adult and elderly cancer survivors. Other study objectives were to (1) investigate the influence of AYA characteristics on IOC and (2) examine the association between IOC and HRQoL among AYAs.

\section{Methods}

\section{Setting and population}

This study is part of a longitudinal population-based survey among lymphoma survivors registered within the
Netherlands Cancer Registry (NCR) in the South of The Netherlands. The NCR records data of all patients newly diagnosed with cancer in the southern part of The Netherlands, an area with 2.3 million inhabitants. The NCR was used to select all patients diagnosed with NHL and HL between January 1, 1999 and January 1, 2009. We included patients aged $\geq 18$ years at time of diagnosis, with all subtypes of indolent (including chronic lymphocytic leukaemia-like) and aggressive B cell NHL and HL as defined by the International Classification of Diseases for Oncology-3 codes (ICD-O3) [22]. Deceased patients were excluded by linking the NCR database with the Central Bureau for Genealogy. Ethical approval for the study was obtained from the local certified Medical Ethics Committee of the Maxima Medical Centre Veldhoven.

\section{Data collection}

Data collection took place in 2009 and was done within PROFILES [23]. PROFILES is a registry for the study of the physical and psychosocial impact of cancer and its treatment from a dynamic, growing population-based cohort of both short- and long-term cancer survivors. PROFILES is linked directly to clinical data from NCR. Details of the data collection method have been previously described [23]. Data from the PROFILES registry are available for noncommercial scientific research, subject to study question, privacy and confidentiality restrictions, and registration (www. profilesregistry.nl).

In May 2009, patients between 6 months and 10 years after diagnosis were included in the study. In November 2009, patients diagnosed between May and August 2009 were also invited to participate to broaden the number of short-term survivors.

\section{Study measures}

\section{Sociodemographic and clinical characteristics}

Clinical information was available from the NCR that routinely collects data on tumour characteristics, primary treatment and patients' background characteristics including sex and date of birth. Comorbidity at time of survey was assessed using a modified version of the Self-Administered Comorbidity Questionnaire assessing the prevalence of 14 comorbidities including heart disease, stroke, high blood pressure, COPD/asthma, diabetes, stomach disease, kidney disease, liver disease, anaemia, depression, thyroid disease, osteoarthritis, back pain, and rheumatoid arthritis [24]. Selfdesigned questions on educational level and marital status were added to the questionnaire. 


\section{Impact of cancer}

The 41-item IOCv1 was used in this study to measure specific positive and negative IOC among long-term cancer survivors $[9,25]$. Patients could indicate their level of agreement on each statement from 1 (strongly disagree) to 5 (strongly agree). The questionnaire was missing one item of the 'negative self-evaluation' subscale by mistake. This missing item was imputed by the mean score of the "negative self-evaluation' subscale for each participant separately. A more recent and comprehensive scaling of the IOCv1 questionnaire yielded the 37-item IOCv2 [26]. A valid algorithm was used to convert the IOCv1 item responses into IOCv2 item scores [27]. Two main summary IOC scales could be formed, a positive $(\alpha=0.74)$ and a negative $(\alpha=0.82)$. The total scale scores were calculated by averaging the item scores. Higher scores on the positive and negative impact summary scales indicate greater positive and negative impacts of cancer, respectively. The two summary scales could be further subdivided into eight subscales. The four positive subscales comprise: altruism/empathy, health awareness, meaning of cancer and positive self-evaluation. The negative subscales are appearance concerns, body change concerns, life interferences and worry.

\section{Health-related quality of life}

The validated Dutch version of the EORTC QLQ-C30 was used to assess HRQoL. The questionnaire includes five scales on physical $(\alpha=0.79)$, role $(\alpha=0.89)$, emotional $(\alpha=0.90)$, cognitive $(\alpha=0.73)$ and social functioning $(\alpha=0.79)$; and a global health status/quality of life scale $(\alpha=0.92)$. Answer categories range from 1 (not at all) to 4 (very much). After linear transformation, all scales range in score from 0 to 100 . A higher score means a better HRQoL [28].

\section{Statistical analysis}

Analyses were performed using Statistical Package for the Social Sciences version 22 (SPSS), Chicago, IL, USA and two-sided $p$ values of $<0.05$ were considered statistically significant. Differences in demographic and clinical characteristics between respondents, non-respondents and cancer survivors with unverifiable addresses and between the three age groups (AYA, adults, elderly) were compared using chisquare and analysis of variance (ANOVA), where appropriate.

ANOVAs were performed to investigate mean differences between the age categories of lymphoma survivors (independent variable) and the IOCv2 total and subscale scores (dependent variables). To counteract the problem of multiple comparisons (type-1 error), Bonferroni correction was used $(p<0.001)$.
Multiple linear regression analyses were used to compare AYA lymphoma survivor characteristics (independent variables) and the mean IOCv2 summary and subscale scores (dependent variables).

Additionally, multiple linear regression analyses were performed to investigate the independent association between IOCv2 total and subscales, and the HRQoL subscales for the AYA sample. All demographic and clinical variables were included as confounders; this was determined a priori based on hypotheses.

\section{Results}

\section{Clinical and sociodemographic characteristics}

Of the 1281 Dutch lymphoma survivors who met the eligibility criteria and received a questionnaire, 861 (67\% response rate) completed it.

A comparison of respondents, non-respondents and survivors with unverifiable addresses, indicated that nonrespondents were older, more recently diagnosed or less often diagnosed with stage I disease, when compared to survivors with unverifiable addresses and respondents.

Of all respondents, $11 \%$ was AYA (18-35 years at diagnosis), 59\% adult (36-64 years at diagnosis) and 30\% elderly (65-84 years at diagnosis).

AYA survivors were more often diagnosed with HL and more often with stage II disease and treated more often with a combination of chemotherapy and radiotherapy, when compared to older lymphoma survivors. Additionally, AYAs reported fewer comorbid conditions, were married less often, and more often had a higher educational level and a job compared to adult and elderly lymphoma survivors (Table 1).

\section{Associations between age and IOC}

Significant differences in IOC between the age groups were observed on the positive impact summary scale $\left(\mathrm{F}(2798)=11.54, p<0.001, \mathrm{eta}^{2}=0.03\right)$ and on the positive subscales 'health awareness' $(p<0.001)$, 'meaning of cancer' $(p=0.001)$ and 'positive self-evaluation' $(p<0.001)$. No significant differences between the age groups were found on negative IOC (Table 2). Sub analyses for HL survivors only showed the same mean scale scores only statistical significance of these findings could not be determined due to the small number of elderly with HL ( $n=10$; data not shown).

\section{Associations between AYA characteristics and IOC}

Female AYAs scored significantly higher on the negative impact summary scale and on the subscales 'appearance concerns' and 'positive self-evaluation' compared to AYA males. 
Table 1 Sociodemographic and clinical characteristics of cancer survivors stratified by age

\begin{tabular}{|c|c|c|c|c|}
\hline & $\begin{array}{l}\text { AYA } \\
N=98\end{array}$ & $\begin{array}{l}\text { Adults } \\
N=507\end{array}$ & $\begin{array}{l}\text { Elderly } \\
N=256\end{array}$ & $p$ value \\
\hline Age (at time of diagnosis) (mean $\pm \mathrm{SD}$ ) & $28.0(5.4)$ & $53.4(8.0)$ & $71.4(4.0)$ & $<0.01$ \\
\hline Age (at time of survey) $($ mean $\pm \mathrm{SD})$ & $32.9(6.0)$ & $58.5(8.2)$ & $75.7(4.0)$ & $<0.01$ \\
\hline Tumour type & & & & $<0.01$ \\
\hline NHL & $33(34 \%)$ & $435(86 \%)$ & $245(96 \%)$ & \\
\hline $\mathrm{HL}$ & $65(66 \%)$ & $71(14 \%)$ & $10(4 \%)$ & \\
\hline Years since diagnosis (mean $\pm \mathrm{SD}$ ) & $5.0(2.5)$ & $5.1(2.6)$ & $4.3(2.4)$ & $<0.01$ \\
\hline Years since diagnosis & & & & $<0.01$ \\
\hline$<2$ years & $12(12 \%)$ & $68(14 \%)$ & $45(18 \%)$ & \\
\hline $2-5$ years & $39(40 \%)$ & $191(38 \%)$ & $128(51 \%)$ & \\
\hline $5-10$ years & $47(48 \%)$ & $233(46 \%)$ & $76(30 \%)$ & \\
\hline$>10$ years & $0(0 \%)$ & $11(2 \%)$ & $4(2 \%)$ & \\
\hline Sex & & & & 0.08 \\
\hline Male & $49(50 \%)$ & $306(60 \%)$ & $161(63 \%)$ & \\
\hline Female & $49(50 \%)$ & $200(40 \%)$ & $94(37 \%)$ & \\
\hline Stage at diagnosis & & & & $<0.01$ \\
\hline I & $19(19 \%)$ & $120(24 \%)$ & $64(25 \%)$ & \\
\hline II & $49(50 \%)$ & $92(18 \%)$ & $42(17 \%)$ & \\
\hline III & $19(19 \%)$ & $71(14 \%)$ & $30(12 \%)$ & \\
\hline IV & $10(10 \%)$ & $121(24 \%)$ & $59(23 \%)$ & \\
\hline Unknown & $1(1 \%)$ & $102(20 \%)$ & $60(24 \%)$ & \\
\hline Primary treatment & & & & $<0.01$ \\
\hline Radiotherapy alone & $5(5 \%)$ & $43(9 \%)$ & $23(9 \%)$ & \\
\hline Chemotherapy alone & $38(39 \%)$ & $227(46 \%)$ & $125(50 \%)$ & \\
\hline Chemotherapy + radiotherapy & $54(55 \%)$ & $98(20 \%)$ & $32(13 \%)$ & \\
\hline Active surveillance ${ }^{a}$ & $0(0 \%)$ & $121(24 \%)$ & $66(26 \%)$ & \\
\hline Surgery & $1(0.5 \%)$ & $5(0.7 \%)$ & $0(0 \%)$ & \\
\hline Transplantation & $0(0 \%)$ & $1(0.2 \%)$ & $0(0 \%)$ & \\
\hline Other therapies & $1(1 \%)$ & $4(1 \%)$ & $6(2 \%)$ & \\
\hline Comorbidity & & & & $<0.01$ \\
\hline None & $63(64 \%)$ & $187(37 \%)$ & $65(26 \%)$ & \\
\hline 1 & $21(21 \%)$ & $154(30 \%)$ & $70(28 \%)$ & \\
\hline 2 & $8(8 \%)$ & $83(16 \%)$ & $63(25 \%)$ & \\
\hline 3 or more & $6(6 \%)$ & $82(16 \%)$ & $57(22 \%)$ & \\
\hline Marital status & & & & $<0.01$ \\
\hline Married/cohabiting & $66(68 \%)$ & $426(85 \%)$ & $181(74 \%)$ & \\
\hline Divorced/separated & $11(11 \%)$ & $29(6 \%)$ & $12(5 \%)$ & \\
\hline Widowed & $1(1 \%)$ & $28(6 \%)$ & $42(17 \%)$ & \\
\hline Never married/never cohabiting & $19(20 \%)$ & $19(4 \%)$ & $11(5 \%)$ & \\
\hline Education level & & & & $<0.01$ \\
\hline Low & $1(1 \%)$ & $57(11 \%)$ & $64(26 \%)$ & \\
\hline Medium & $54(57 \%)$ & $314(63 \%)$ & $138(56 \%)$ & \\
\hline High & $40(42 \%)$ & $129(26 \%)$ & $43(18 \%)$ & \\
\hline Current occupation & & & & $<0.01$ \\
\hline Employed & $64(74 \%)$ & $173(37 \%)$ & $2(1 \%)$ & \\
\hline Not working/retired & $22(26 \%)$ & $296(63 \%)$ & $244(99 \%)$ & \\
\hline
\end{tabular}

AYA (18-35 years), Adults (36-64 years), Elderly (65-84 years): age at time of diagnosis

NHL non-Hodgkin lymphoma, HL Hodgkin lymphoma education levels included low no/primary school, medium lower general secondary education/vocational training, or high pre-university education/high vocational training/ university

${ }^{a}$ Patients under active surveillance and receive no therapy 
Table 2 Mean scores of the Impact of Cancer (IOCv2) sub and total scales $( \pm \mathrm{SD})$ according to age

\begin{tabular}{lllll}
\hline & AYA & Adults & Elderly & $p$ value \\
& Me98 & $N=507$ & $N=256$ & \\
IOCv2 & & Mean (SD) & Mean (SD) & \\
\hline Altruism/empathy & $3.3(0.7)$ & $3.2(0.8)$ & $3.2(0.9)$ & 0.64 \\
Health awareness & $3.5(0.9)$ & $3.3(0.8)$ & $3.0(1.0)$ & $<0.001^{\mathrm{a}}$ \\
Meaning of cancer & $2.8(0.8)$ & $2.7(0.8)$ & $2.5(0.9)$ & $0.001^{\mathrm{a}}$ \\
Positive self-evaluation & $3.8(0.7)$ & $3.4(0.8)$ & $3.2(0.9)$ & $<0.001^{\mathrm{b}}$ \\
Appearance concerns & $1.8(1.0)$ & $1.8(0.8)$ & $1.8(0.8)$ & 0.94 \\
Body change concerns & $2.6(1.1)$ & $2.6(1.0)$ & $2.6(1.0)$ & 0.51 \\
Life interferences & $1.9(0.5)$ & $2.0(0.5)$ & $2.0(0.5)$ & 0.09 \\
Worry & $2.6(0.9)$ & $2.8(0.9)$ & $2.6(1.0)$ & 0.005 \\
Positive impact scale & $3.4(0.6)$ & $3.1(0.6)$ & $3.0(0.7)$ & $<0.001^{\mathrm{c}}$ \\
Negative impact scale & $2.2(0.7)$ & $2.3(0.7)$ & $2.2(0.7)$ & 0.19 \\
\hline
\end{tabular}

Bonferonni correction $p=0.01 / 10=<0.001$

IOCV2 Impact of Cancer Scale version 2: high scores representing greater impact

AYA (18-35 years), Adults (36-64 years), Elderly (65-84 years)

${ }^{\text {a }}$ Between AYA and elderly and between adults and elderly

${ }^{\mathrm{b}}$ Between AYA and adults and between AYA and elderly

${ }^{\mathrm{c}}$ Between AYA and adults, between AYA and elderly and between adults and elderly

AYA survivors with a partner scored higher on the negative impact summary scale and 'body change concerns'. AYAs with more comorbidities reported more impact on 'appearance concerns' and AYA survivors with elevated psychological distress scored significantly higher on all IOCv2 scales, except for the positive impact summary scale and the subscales 'meaning of cancer' and 'positive self-evaluation' (Table 3).

\section{Associations between IOC and HRQoL among AYA lymphoma survivors}

After adjusting for sociodemographic and clinical factors, the negative impact summary scale was negatively associated with all HRQoL scales (Table 4). The positive impact summary scale was negatively associated with the EORTC QLQC30 subscale 'Emotional functioning', which was mainly caused by the items of the IOC subscales 'altruism/empathy' and 'health awareness'.

\section{Discussion}

This study showed that AYA, adult and elderly (non-)Hodgkin lymphoma survivors experienced different types of positive and negative IOC. Our results are in line with those of previous studies among lymphoma survivors, which showed that younger survivors had higher positive impact scores compared to older survivors $[11,29]$. The positive IOC among
AYAs is reflected in more 'health awareness' (e.g. better self-care), 'meaning of cancer' (e.g. giving direction in life) and 'positive self-evaluation' (e.g. considering oneself to be a role model). An explanation could be that AYAs make use of more active and adaptive coping mechanisms and might be more resilient compared to older cancer survivors [30-33]. Resilience is described as a personality profile characterized by 'positive adaptation' after a stressful life event [34, 35]. Moreover, studies show that younger cancer survivors report higher levels of post-traumatic growth [36, 37], which refers to a set of positive changes occurring as a result of coping with a traumatic event like cancer. AYAs might experience more personal growth after their cancer experience compared to older cancer survivors as they are more likely to give direction in life and report adequate health competence after a cancer experience [38]. On the other hand, contrary to cancer survivors, healthy older adults are more resilient than young adults [39].

In contrast to previous studies [5, 11, 29], showing that younger cancer survivors had higher negative impact scores when compared to older survivors, our results showed no significant differences for negative impacts of cancer between the three age groups. One possible explanation could be that the majority of lymphoma survivors did not undergo invasive or surgical treatments and they experienced, for example, less 'body change concerns' or 'appearance concerns' compared to survivors treated for other types of cancer. Another explanation could be that the IOC questionnaire lacks important age-specific concerns. Development of more age-specific questionnaires will make it possible to accurately identify IOC and prioritize the perceived age-specific needs of cancer survivors $[40,41]$.

Female AYAs scored significantly higher on the negative IOC, compared to young men. This gender difference is largely consistent with previous studies on long-term cancer survivors of all ages [5, 42]. A possible explanation could be the presence of higher rates of psychological distress in the female population in general, as opposed to men [43, 44]. This sex difference may reflect a difference in willingness to report distress among men, but could also occur because women tend to use more emotional coping styles [45-47]. The negative IOC among female AYAs is reflected in more 'appearance concerns' compared to men. This is in line with the literature which shows that especially young women, report more experiences associated with cancer-related appearance and body image concerns, than young men cancer survivors [48]. For example, women are more aware of their looks, and may feel more disfigured than men. On the other hand, female AYAs reported higher scores on the positive subscale 'positive self-evaluation'. This means that young women consider themselves as a cancer survivor and/or role model, and may feel a sense of pride from surviving cancer. A possible explanation for this finding could be the tendency of women to 
Table 3 Multiple linear regression analyses of IOCv2 total and subscales of AYA lymphoma survivors $(N=98)$

\begin{tabular}{|c|c|c|c|c|c|c|c|c|c|c|}
\hline & \multicolumn{5}{|c|}{ Positive subscales } & \multicolumn{5}{|c|}{ Negative subscales } \\
\hline & $\begin{array}{l}\text { Positive } \\
\text { impact }\end{array}$ & $\begin{array}{l}\text { Altruism/ } \\
\text { empathy }\end{array}$ & $\begin{array}{l}\text { Health } \\
\text { awareness }\end{array}$ & $\begin{array}{l}\text { Meaning } \\
\text { of } \\
\text { cancer }\end{array}$ & $\begin{array}{l}\text { Positive } \\
\text { self- } \\
\text { evaluation }\end{array}$ & $\begin{array}{l}\text { Negative } \\
\text { impact }\end{array}$ & $\begin{array}{l}\text { Appearance } \\
\text { concerns }\end{array}$ & $\begin{array}{l}\text { Body } \\
\text { change } \\
\text { concerns }\end{array}$ & $\begin{array}{l}\text { Life } \\
\text { interferences }\end{array}$ & Worry \\
\hline & Bèta & Bèta & Bèta & Bèta & Bèta & Bèta & Bèta & Bèta & Bèta & Bèta \\
\hline $\begin{array}{l}\text { Age (at time of } \\
\text { survey) }\end{array}$ & 0.05 & 0.04 & 0.13 & -0.02 & -0.08 & 0.00 & 0.06 & -0.04 & -0.04 & 0.11 \\
\hline \multicolumn{11}{|l|}{ Tumour type } \\
\hline NHL & Ref & Ref & Ref & Ref & Ref & Ref & Ref & Ref & Ref & Ref \\
\hline $\mathrm{HL}$ & -0.03 & -0.06 & 0.10 & -0.10 & 0.06 & -0.13 & -0.17 & -0.03 & -0.11 & -0.02 \\
\hline \multicolumn{11}{|l|}{$\begin{array}{l}\text { Years since } \\
\text { diagnosis }\end{array}$} \\
\hline$\leq 5$ years & Ref & Ref & Ref & Ref & Ref & Ref & Ref & Ref & Ref & Ref \\
\hline$>5$ years & 0.14 & 0.19 & 0.04 & 0.10 & 0.03 & -0.01 & -0.19 & 0.08 & -0.06 & 0.03 \\
\hline \multicolumn{11}{|l|}{ Sex } \\
\hline Male & Ref & Ref & Ref & Ref & Ref & Ref & Ref & Ref & Ref & Ref \\
\hline Female & 0.10 & -0.10 & 0.21 & -0.03 & $0.30 *$ & $0.22 *$ & $0.21 *$ & 0.13 & 0.15 & 0.11 \\
\hline \multicolumn{11}{|l|}{ Education } \\
\hline Low & -0.01 & 0.11 & 0.05 & -0.14 & -0.06 & 0.04 & -0.06 & 0.04 & -0.01 & 0.10 \\
\hline Medium & -0.02 & -0.12 & -0.00 & -0.13 & 0.18 & 0.14 & 0.14 & 0.18 & 0.11 & 0.08 \\
\hline High & Ref & Ref & Ref & Ref & Ref & Ref & Ref & Ref & Ref & Ref \\
\hline \multicolumn{11}{|c|}{ Current occupation } \\
\hline Employed & Ref & Ref & Ref & Ref & Ref & Ref & Ref & Ref & Ref & Ref \\
\hline $\begin{array}{l}\text { Not working/ } \\
\text { retired }\end{array}$ & -0.06 & -0.01 & -0.11 & 0.17 & -0.25 & 0.09 & 0.16 & 0.10 & 0.12 & -0.04 \\
\hline \multicolumn{11}{|l|}{ Marital status } \\
\hline No partner & Ref & Ref & Ref & Ref & Ref & Ref & Ref & Ref & Ref & Ref \\
\hline Partner & 0.05 & 0.10 & 0.11 & -0.07 & -0.03 & $0.22 *$ & 0.15 & $0.24 *$ & 0.16 & 0.14 \\
\hline \multicolumn{11}{|l|}{ Comorbidity } \\
\hline No & Ref & Ref & Ref & Ref & Ref & Ref & Ref & Ref & Ref & Ref \\
\hline Yes & 0.07 & 0.03 & 0.02 & 0.01 & 0.08 & 0.15 & $0.24 *$ & 0.17 & 0.08 & 0.06 \\
\hline \multicolumn{11}{|c|}{ Psychological distress (HADS) } \\
\hline$\leq 15$ & Ref & Ref & Ref & Ref & Ref & Ref & Ref & Ref & Ref & Ref \\
\hline$>15$ & 0.24 & $0.28^{*}$ & $0.29 *$ & 0.00 & 0.12 & $0.44 * *$ & $0.31 * *$ & $0.41^{* *}$ & $0.49 * *$ & $0.31 * *$ \\
\hline
\end{tabular}

NHL non-Hodgkin lymphoma, HL Hodgkin lymphoma; Education levels included low $=$ no/primary school, medium $=$ lower general secondary education/vocational training, or high $=$ pre-university education/high vocational training/university

HADS Hospital Anxiety Depression Scale

${ }^{*} p<0.05 ;{ }^{* *} p<0.01$

score higher on post-traumatic growth. This explanation suggests that men and women differ in their responses to a traumatic (cancer) experience [49]. Moreover, an American study found that the post-traumatic growth scores of severely traumatized women were twice as high as those of traumatized men. It suggests that these events have greater effect on women, in that women may be more capable than men of learning or benefiting from difficult life experiences like surviving cancer [49].

The majority of AYA cancer survivors in this study reported that having more psychological distress in general life results in higher scores on the negative IOC summary scale.
This was reflected in more 'appearance concerns', 'body change concerns', 'life interferences' and 'worry'. On the other hand, more psychological distress had a more positive impact on the positive subscales 'altruism/empathy' and 'health awareness'. One possible explanation for this conflicting finding could be that cultural differences between lymphoma survivors living in the USA (where the IOC questionnaire was developed) and Dutch lymphoma survivors affected the IOC scores [11]. Living in different cultures creates other psychological resources that influence health. Since the sense of personal control is more prevalent in North America than in Europe, American survivors are more likely to alter 


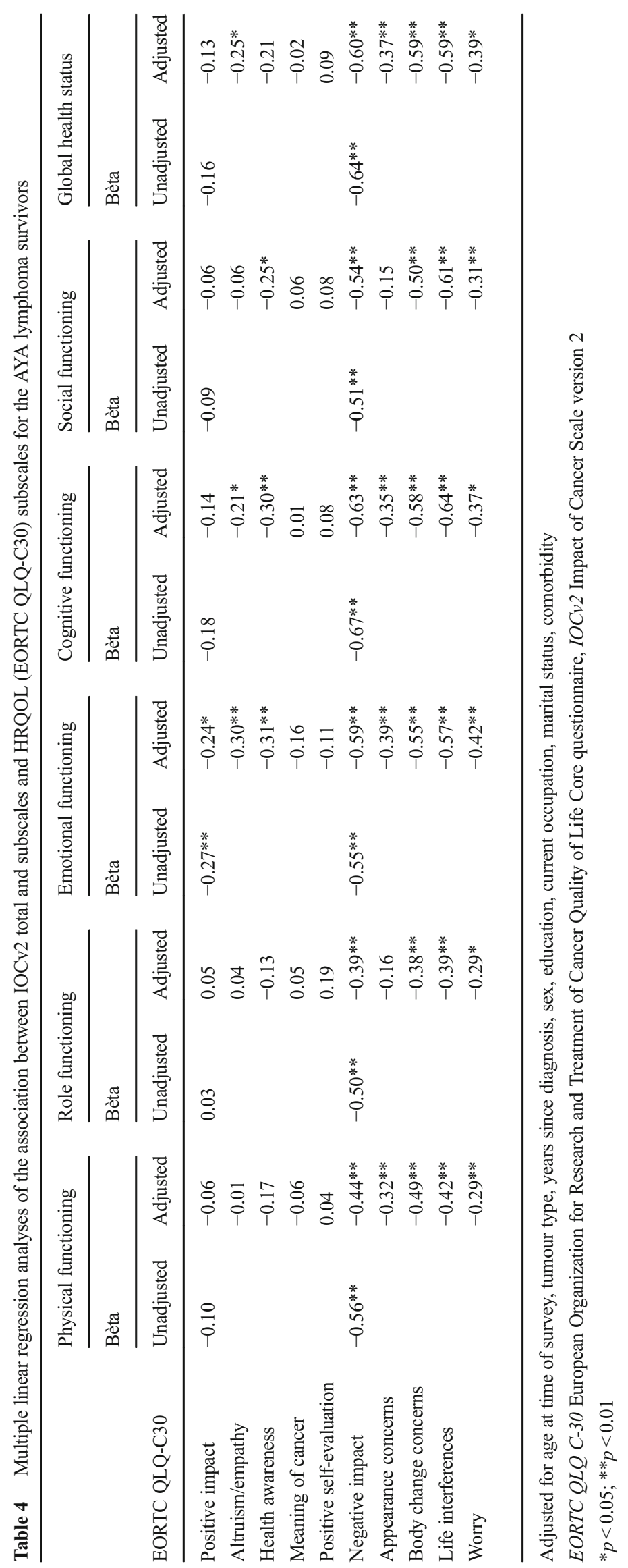


perceptions of the cancer experience in a more positive way [50]. To give an example, the subscale 'health awareness' is seen as something positive in the USA, while in The Netherlands, we may experience it as something more negative [11]. Moreover, the conflicting finding that AYAs with elevated levels of distress experience both negative and positive IOC may indicate the coexistence of the unique negative and positive influences of cancer further in life. This gives reason to belief that mental health is not a single continuum with negative impacts (distress) on one, and positive impacts (growth) on the other side, but rather represents a bivariate construct with two separate dimensions [51].

AYA cancer survivors with a partner scored significantly higher on the negative IOC summary scale and the subscale 'body change concerns', compared to AYAs without a partner. This finding seems very age-related, as previous studies on the general cancer population of all ages show that having a partner reduces the negative impacts of cancer [5, 21]. A first possible explanation could be that AYAs are in a vulnerable phase of life, in which intimate and sexual relationships are evolving. Especially cancer residual symptoms like 'body change concerns' and altered body image could have a more negative impact on AYAs with a partner when compared to AYAs without a partner [52]. Feelings of being unattractive, different or imperfect, or possible impairments in social skills could be barriers for a romantic relationship and may lead to more negative impacts of cancer [53]. Second, AYAs are in a phase of life in which plans for having children may arise. Especially AYAs with a partner may worry about infertility concerns after their cancer treatment, more than AYAs without a partner. Previous research shows that more than fifty percent of AYAs with a partner reported that their cancer experience had a negative impact on their plans for having children [21]. Third, AYA cancer survivors with a partner may experience feelings of guilt and shortcoming, because they "dragged" their partner into a medical situation with an uncertain future. Discordant feelings between the AYA cancer survivors and their partner could arise and have a negative impact on the survivors life [54]. These factors may contribute to higher negative IOC scores among AYAs with a partner.

Consistent with other studies [25], our findings indicate that an individual's perception of the positive and negative IOC on various life domains is related to their functional and global health status. Especially the negative IOC is of influence and is significantly associated with HRQoL. These findings provide evidence for the notion that negative experiences may be more heavily related to overall well-being and functioning than positive experiences of cancer among AYAs. A possible explanation could be the fact that perceived negative outcomes may be more heavily weighted in determining psychological adjustment than positive change [55]. Additionally, as female AYAs, AYAs with elevated levels of distress, and AYAs with a partner reported higher levels of negative IOC, they are considered a "high-risk" population of having a lower HRQoL. Especially these groups of AYA lymphoma survivors should be screened for their HRQoL and should be more intensively guided by health care practitioners.

This study has a few limitations. First, although information was present concerning demographic and clinical characteristics of the non-respondents and survivors with unverifiable addresses, it remains unknown why non-respondents declined to participate in the study. Secondly, it cannot be ruled out that part of the differences in IOC subscale scores between the age groups are a result of the differences in tumour types diagnosed (and accompanied treatment regimens) between younger and older patients. Older patients more often have indolent NHL with a wait and see policy (and therefore probably more late effects of the cancer itself), while younger patients more often have aggressive NHL or HL and therefore receive a combination of chemo- and radiotherapy. However, sub analyses for HL survivors only showed the same mean IOC scale scores as for the total lymphoma sample, indicating that our results are mainly caused by age. Third, the crosssectional design of our study limits the determination of causal associations between the study variables. Fourth, although the IOCv1 and v2 scales showed good psychometric properties among the whole age range, both do not include domains related to developmental issues (e.g. lagging behind healthy peers; difficulties to return to school or problems with getting a job) which are particularly important for AYAs. Fifth, no data were available of healthy AYAs as the IOC is a cancerspecific measure. The question remains whether young people in general experience more positive outcomes than older people or that our results indicate a cancer-specific effect of age [39]. Sixth, the study results cannot be generalized for the total AYA population as Hodgkin's disease has a relatively good prognosis, what may give a more positive indication, compared to other types of cancer.

To promote the positive IOC among AYAs, future research in the area of cognitive and behavioural interventions (e.g. cognitive re-framing) is needed to determine if positive reinterpretation of a negative traumatic event could subsequently lead to adequate resilience skills and coping mechanisms, more positive growth, improved HRQoL and thereby possibly indirectly diminishing the negative IOC. For example, to improve the coping mechanisms of AYAs, psychological interventions directed at positive reframing of cancer should see the cancer experience as an opportunity to negotiate new challenges, re-evaluate life goals and priorities, and enhance selfknowledge may be particularly relevant and effective, especially for younger survivors. Additionally, recommended psychosocial intervention programs could include among others positive peer support, as well as psychoeducational interventions and therapies that focus on engaging cancer patients and facilitating change, by encouraging patients' flexibility to accept what cannot be altered and committing themselves to 
what can be achieved (e.g. 'Acceptance and Commitment Therapy') [56-58]. On the other hand, to diminish or prevent negative IOC, health care providers may offer age-appropriate information to AYAs [56, 59], which may help them to get a more coherent understanding of their illness [51, 60, 61].

To conclude, this study highlights the importance of the specific IOC of AYAs according to their life phase, as compared to adults and the elderly lymphoma survivors. To address the unique needs of AYAs, research should focus on developing questionnaires and interventions more coherent to the age of the cancer survivor. Randomized intervention studies with large samples that focus on psychosocial outcomes are needed to establish evidence-based psycho-oncological interventions for AYAs. Healthcare practitioners need to become aware of the specific age-related impacts of cancer and developmental issues that AYA, adult and elderly lymphoma survivors are dealing with, even years after diagnosis. By keeping this in mind, healthcare practitioners can contribute to a better HRQoL and a more adaptive and supportive healthcare system for the lymphoma survivors.

Compliance with ethical standards All procedures performed in studies involving human participants were in accordance with the ethical standards of the institutional and/or national research committee and with the 1964 Helsinki Declaration and its later amendments or comparable ethical standards.

Conflict of interest The authors declare that they have no competing interests.

Informed consent Informed consent was obtained from all individual participants included in the study.

Open Access This article is distributed under the terms of the Creative Commons Attribution 4.0 International License (http:// creativecommons.org/licenses/by/4.0/), which permits unrestricted use, distribution, and reproduction in any medium, provided you give appropriate credit to the original author(s) and the source, provide a link to the Creative Commons license, and indicate if changes were made.

\section{References}

1. Cijfers over kanker. 2015. Available: http://www.cijfersoverkanker. nl. Accessed 17 November 2015.

2. Jemal A, Bray F, Center MM, Ferlay J, Ward E, Forman D. Global cancer statistics. CA Cancer J Clin. 2011;61(2):69-90.

3. Kluin-Nelemans J, Schot B, Mulder A. Overzicht diagnostieklaboratoriumonderzoek en beeldvorming. Hematologie: Springer. 2013. p. 221-32.

4. Sant M, Minicozzi P, Mounier M, Anderson LA, Brenner H, Holleczek B, et al. Survival for haematological malignancies in Europe between 1997 and 2008 by region and age: results of EUROCARE-5, a population-based study. Lancet Oncol. 2014;15(9):931-42.

5. Korszun A, Sarker SJ, Chowdhury K, Clark C, Greaves P, Johnson $\mathrm{R}$, et al. Psychosocial factors associated with impact of cancer in longterm haematological cancer survivors. Br J Haematol. 2014;164(6):790-803.

6. Geffen DB, Blaustein A, Amir MC, Cohen Y. Post-traumatic stress disorder and quality of life in long-term survivors of Hodgkin's disease and non-Hodgkin's lymphoma in Israel. Leuk Lymphoma. 2003;44(11):1925-9.

7. Leukemia \& Lymphoma, Society. Long-term and late effects of treatment in adults facts. 2012.

8. Allart P, Soubeyran P, Cousson-Gélie F. Are psychosocial factors associated with quality of life in patients with haematological cancer? A critical review of the literature. Psycho-Oncology. 2013;22(2):241-9.

9. Zebrack BJ, Ganz PA, Bernaards CA, Petersen L, Abraham L. Assessing the impact of cancer: development of a new instrument for long-term survivors. Psychooncology. 2006;15(5):407-21.

10. van der Poel MW, Oerlemans S, Schouten HC, Mols F, Pruijt JF, Maas H, et al. Quality of life more impaired in younger than in older diffuse large B cell lymphoma survivors compared to a normative population: a study from the population-based PROFILES registry. Ann Hematol. 2014;93(5):811-9.

11. Oerlemans S, Smith SK, Crespi CM, Zimmerman S, van de PollFranse LV, Ganz PA. Assessing the impact of cancer among Dutch non-Hodgkin lymphoma survivors compared with their American counterparts: a cross-national study. Psychooncology. 2013;22(6): $1258-65$.

12. Rowland JH. Developmental stage and adaptation: adult model 1989. 25-45 p.

13. Hurria A, Li D, Hansen K, Patil S, Gupta R, Nelson C, et al. Distress in older patients with cancer. J Clin Oncol. 2009;27(26): 4346-51.

14. Reeve BB, Potosky AL, Smith AW, Han PK, Hays RD, Davis WW, et al. Impact of cancer on health-related quality of life of older Americans. J Natl Cancer Inst. 2009;101(12):860-8.

15. Blank TO, Bellizzi KM. A gerontologic perspective on cancer and aging. Cancer. 2008;112(S11):2569-76.

16. Mols F, Thong MS, Vreugdenhil G, van de Poll-Franse LV. Longterm cancer survivors experience work changes after diagnosis: results of a population-based study. Psychooncology. 2009;18(12):1252-60.

17. Mellon S, Northouse LL. Family survivorship and quality of life following a cancer diagnosis. Res Nurs Health. 2001;24(6):446-59.

18. Suris J-C, Michaud P-A, Viner R. The adolescent with a chronic condition. Part I: developmental issues. Arch Dis Child. 2004;89(10):938-42.

19. Nass SJ, Patlak M. Identifying and addressing the needs of adolescents and young adults with cancer: workshop summary. 2014.

20. Foster RH, Stern M. Peer and romantic relationships among adolescent and young adult survivors of childhood hematological cancer: a review of challenges and positive outcomes. Acta Haematol. 2014;132(3-4):375-82.

21. Bellizzi KM, Smith A, Schmidt S, Keegan TH, Zebrack B, Lynch $\mathrm{CF}$, et al. Positive and negative psychosocial impact of being diagnosed with cancer as an adolescent or young adult. Cancer. 2012;118(20):5155-62.

22. Fritz AG. International classification of diseases for oncology: ICDO: World Health Organization. 2000.

23. van de Poll-Franse LV, Horevoorts N, van Eenbergen M, Denollet J, Roukema JA, Aaronson NK, et al. The patient reported outcomes following initial treatment and long term evaluation of survivorship registry: scope, rationale and design of an infrastructure for the study of physical and psychosocial outcomes in cancer survivorship cohorts. Eur J Cancer. 2011;47(14):2188-94.

24. Sangha O, Stucki G, Liang MH, Fossel AH, Katz JN. The selfadministered comorbidity questionnaire: a new method to assess comorbidity for clinical and health services research. Arthritis Care Res. 2003;49(2):156-63. 
25. Zebrack BJ, Yi J, Petersen L, Ganz PA. The impact of cancer and quality of life for long-term survivors. Psycho-Oncology. 2008;17(9):891-900.

26. Crespi CM, Ganz PA, Petersen L, Castillo A, Caan B. Refinement and psychometric evaluation of the impact of cancer scale. J Natl Cancer Inst. 2008;100(21):1530-41.

27. Crespi CM, Ganz PA, Petersen L, Smith SK. A procedure for obtaining impact of cancer version 2 scores using version 1 responses. Qual Life Res. 2013;22(1):103-9.

28. Aaronson NK, Ahmedzai S, Bergman B, Bullinger M, Cull A, Duez NJ, et al. The European organization for research and treatment of cancer QLQ-C30: a quality-of-life instrument for use in international clinical trials in oncology. J Natl Cancer Inst. 1993;85(5):365-76.

29. Smith SK, Crespi CM, Petersen L, Zimmerman S, Ganz PA. The impact of cancer and quality of life for post-treatment non-Hodgkin lymphoma survivors. Psychooncology. 2010;19(12):1259-67.

30. Molina Y, Jean CY, Martinez-Gutierrez J, Reding KW, Joyce P, Rosenberg AR. Resilience among patients across the cancer continuum: diverse perspectives. Clin J Oncol Nurs. 2014;18(1):93.

31. Campbell-Sills L, Cohan SL, Stein MB. Relationship of resilience to personality, coping, and psychiatric symptoms in young adults. Behav Res Ther. 2006;44(4):585-99.

32. Woodgate RL. A review of the literature on resilience in the adolescent with cancer: Part II. J Pediatr Oncol Nurs. 1999;16(2):7889.

33. Hilton BA. The relationship of uncertainty, control, commitment, and threat of recurrence to coping strategies used by women diagnosed with breast cancer. J Behav Med. 1989;12(1):39-54.

34. Robins RW, John OP, Caspi A, Moffitt TE, Stouthamer-Loeber M. Resilient, overcontrolled, and undercontrolled boys: three replicable personality types. J Pers Soc Psychol. 1996;70(1):157.

35. Asendorpf JB, van Aken MA. Resilient, overcontrolled, and undercontroleed personality prototypes in childhood: replicability, predictive power, and the trait-type issue. J Pers Soc Psychol. 1999;77(4):815.

36. Pudrovska T. What makes you stronger age and cohort differences in personal growth after cancer. J Health Soc Behav. 2010;51(3): 260-73.

37. Widows MR, Jacobsen PB, Booth-Jones M, Fields KK. Predictors of posttraumatic growth following bone marrow transplantation for cancer. Health Psychol. 2005;24(3):266.

38. Zebrack B, Kwak M, Salsman J, Cousino M, Meeske K, Aguilar C, et al. The relationship between posttraumatic stress and posttraumatic growth among adolescent and young adult (AYA) cancer patients. Psycho-Oncology. 2015;24(2):162-8.

39. Gooding P, Hurst A, Johnson J, Tarrier N. Psychological resilience in young and older adults. Int J Geriatr Psychiatry. 2012;27(3):26270.

40. Clinton-McHarg T, Carey M, Sanson-Fisher R, D'Este C, Shakeshaft A. Preliminary development and psychometric evaluation of an unmet needs measure for adolescents and young adults with cancer: the Cancer Needs Questionnaire-Young People (CNQ-YP). Health Qual Life Outcomes. 2012;10:13.

41. Zebrack B, Bleyer A, Albritton K, Medearis S, Tang J. Assessing the health care needs of adolescent and young adult cancer patients and survivors. Cancer. 2006;107(12):2915-23.

42. Holterhues C, Cornish D, van de Poll-Franse LV, Krekels G, Koedijk F, Kuijpers D, et al. Impact of melanoma on patients' lives among 562 survivors: a dutch population-based study. Arch Dermatol. 2011;147(2):177-85.

43. Linden W, Vodermaier A, MacKenzie R, Greig D. Anxiety and depression after cancer diagnosis: prevalence rates by cancer type, gender, and age. J Affect Disord. 2012;141(2):343-51.
44. Wang K, Liu X, Wang L. Age and gender differences in the association between serious psychological distress and cancer: findings from the 2003, 2005, and 2007 Health Information National Trends Surveys (HINTS). Am J Cancer Sci. 2014;3(1):54-66.

45. Goldzweig G, Andritsch E, Hubert A, Walach N, Perry S, Brenner $\mathrm{B}$, et al. How relevant is marital status and gender variables in coping with colorectal cancer? A sample of middle-aged and older cancer survivors. Psycho-Oncology. 2009;18(8):866-74.

46. Jacobs-Lawson JM, Schumacher MM, Hughes T, Arnold S. Gender differences in psychosocial responses to lung cancer. Gend Med. 2010;7(2):137-48.

47. Stanton AL, Danoff-Burg S, Cameron CL, Bishop M, Collins CA, Kirk SB, et al. Emotionally expressive coping predicts psychological and physical adjustment to breast cancer. J Consult Clin Psychol. 2000;68(5):875.

48. Cash TF. Encyclopedia of body image and human appearance: Academic Press. 2012.

49. Tedeschi RG, Calhoun LG. The posttraumatic growth inventory: measuring the positive legacy of trauma. J Trauma Stress. 1996;9(3):455-71.

50. Clarke P, Smith J. Aging in a cultural context: cross-national differences in disability and the moderating role of personal control among older adults in the United States and England. J Gerontol Ser B Psychol Sci Soc Sci. 2011;66(4):457-67.

51. Husson O, Mols F, Oranje WA, Haak HR, Nieuwlaat WA, NeteaMaier RT, et al. Unmet information needs and impact of cancer in (long-term) thyroid cancer survivors: results of the PROFILES registry. Psychooncology. 2014;23(8):946-52.

52. Ussher JM, Perz J, Gilbert E. Women's sexuality after cancer: a qualitative analysis of sexual changes and renegotiation. Women Ther. 2014;37(3-4):205-21.

53. Evan EE, Kaufman M, Cook AB, Zeltzer LK. Sexual health and self-esteem in adolescents and young adults with cancer. Cancer. 2006;107(S7):1672-9.

54. McKenzie H, Crouch M. Discordant feelings in the lifeworld of cancer survivors. Health. 2004;8(2):139-57.

55. Pinquart M, Frohlich C, Silbereisen RK. Cancer patients' perceptions of positive and negative illness-related changes. J Health Psychol. 2007;12(6):907-21.

56. Richter D, Koehler M, Friedrich M, Hilgendorf I, Mehnert A, Weißflog G. Psychosocial interventions for adolescents and young adult cancer patients: a systematic review and meta-analysis. Crit Rev Oncol Hematol. 2015.

57. Zebrack B, Isaacson S. Psychosocial care of adolescent and young adult patients with cancer and survivors. J Clin Oncol. 2012;30(11): $1221-6$.

58. Ruiz FJ. Acceptance and commitment therapy versus traditional cognitive behavioral therapy: a systematic review and metaanalysis of current empirical evidence. Int J Psychol Psychol Ther. 2012;12(3):333-58.

59. Goldfarb M, Casillas J. Unmet information and support needs in newly diagnosed thyroid cancer: comparison of adolescents/young adults (AYA) and older patients. J Cancer Survivorship. 2014;8(3): 394-401.

60. Smith AW, Parsons HM, Kent EE, Bellizzi K, Zebrack BJ, Keel G, et al. Unmet support service needs and health-related quality of life among adolescents and young adults with cancer: the AYA HOPE study. Front Oncol. 20133.

61. Husson O, Thong MS, Mols F, Oerlemans S, Kaptein AA, PollFranse LV. Illness perceptions in cancer survivors: what is the role of information provision? Psycho-Oncology. 2013;22(3):490-8. 